\title{
Rousseau y la incipiente democracia salvadoreña
}

La idea de que los acuerdos de paz constituían una forma de pacto social y de que en El Salvador un futuro democrático y pacífico dependería de la solidez del nuevo consenso nacional alcanzado, ha merecido - desde que fue expresada en Chapultepec el 16 de enero de 1992- una general aceptación y ha sido, desde entonces, innumerables veces repetida. Para muchos, la idea parecía novedosa, y son pocos los que han reparado en su clara conexión con la concepción de contrato social que desarrollase, a mediados del siglo XVIII, un agudo pensador: Juan Jacobo Rousseau.

Por otra parte, desde que terminó el conflicto, la democracia ha levantado tan unánimes adhesiones, que tal pareciera que fuese éste un país lleno de "demócratas de toda la vida". La real sinceridad de dichas posiciones está todavía por verse, pero se reconoce al menos que faltan tradiciones y cultura democrática, donde la democracia pueda echar raíces. Hay conciencia de que la sola reforma de las instituciones no basta; de que se precisa de nuevas prácticas, actitudes, costumbres y valores; de que a un nuevo estilo de hacer política, deberá corresponder un esfuerzo de educación ciudadana de amplio alcance. Se trata, nuevamente, de una problemática típicamente rusoniana - planteada audazmente en 1762 en el Emilio - frente a la que está confrontado El Salvador de nuestros días: la de cómo educar al hombre para la sociedad, la de cómo incidir desde la educación en la comunidad, desde el individuo en la colectividad, desde la ética en la política.

En tercer lugar, cabe decir que, pese al actual entusiasmo ideológico que la democracia despierta por nuestros lares, como si fuera la solución a todos los problemas, las cosas no son tan sencillas. En muchas de las naciones con tradición democrática, sus ciudadanos hace tiempo que "vienen de regreso": sólo la mitad participa en los procesos electorales y, de ellos, según las encuestas, la mitad no cree que aquéllos sirvan para mayor cosa. Mientras en El Salvador el "cansancio de la guerra" tuvo el saludable efecto de contribuir a acelerar la solución negociada, en otros países, el cansancio de la política —el llamado "desencanto"sólo ha aumentado la alienación y el individualismo. Aún más paradójico: si bien en El Salvador la guerra ha traído democracia, en Bosnia, inversamente, podríamos decir que la democracia ha traído la guerra. Por tanto, decir democracia no es suficiente. Habrá que ver sobre qué bases, cuáles sus límites y sus riesgos. En el fondo, sigue palpitando la inquietante pregunta rusoniana, de si es factible la democracia en medio de grandes desigualdades económicas y sociales. ¿Puede, una sociedad como la salvadoreña, volverse más democrática si no se hace, al mismo tiempo, más igualitaria?

En primer lugar, resulta provechoso reflexionar acerca del tipo de contrato que representa el Acuerdo de Paz logrado en El Salvador. En la negociación salvadoreña, a diferencia del contrato rusoniano, ha habido dos partes (FMLN y gobierno) que eran preexistentes y, lo que es más importante, dos partes que juntas no alcanzan a representar a la totalidad de la nación salvadorefía. El hecho de haber dejado excluidas a otras fuerzas 
políticas y a la totalidad de las fuerzas sociales del proceso de negociación ha sido, y sigue siendo, uno de los puntos débiles del acuerdo logrado.

Lógicamente, por haber sido el proceso de elaboración del nuevo contrato un proceso real, que dista mucho de la convención teórica ideal imaginada por Rousseau, no se ha dado tampoco otra de las características de ésta: la inexistencia, por innecesario, de un árbitro. Para el contrato social salvadoreño no solamente ha sido imprescindible un árbitro, sino que además fue necesario encontrar al más cualificado, honorable y poderoso: la Organización de Naciones Unidas.

Ello pone en evidencia la naturaleza conflictiva, no unánime, dificultosa, de la negociación y del acuerdo alcanzado. Se trata de un contrato en el que no todos los que intervienen están íntimamente convencidos de no haber concedido más de lo que han obtenido finalmente. Se está muy lejos, por tanto, de las condiciones ideales del contrato rusoniano, en que es la libre voluntad y el convencimiento de realizar un intercambio mutuamente ventajoso lo que cimenta un auténtico consenso. Ello es lo que ha obligado a que la Naciones Unidas haya tenido que jugar, abiertamente, la función de arbitraje, inicialmente excluida, una vez que tanto la primitiva misión "de buenos oficios", como la posterior "mediación activa", demostraron, en las crisis sucesivas y cada vez más graves, su impotencia frente a la compleja dinámica del proceso de paz.

Debemos matizar también que el hecho de que Naciones Unidas haya sido y siga siendo el árbitro del contrato de Chapultepec y del proceso pacificador, no significa que este tercero intervenga desde afuera del contrato o que no deba ser considerado como una parte interesada. Es al contrario. Fue justamente a los gobiernos de los países con más intereses en la región, a los que se invitó para que tuvieran una participación más destacada. Tanto la conformación del inicial grupo de "países amigos", como el hecho de que, a la hora de entrar en la fase de aplicación de los acuerdos, se añadiera a aquéllos el gobierno de Estados Unidos, conformándose así el "Grupo $4+1$ ", es suficientemente demostrativo de ello. Es falso, por tanto, afirmar que el Acuerdo de Paz es una cuestión que atañe solamente a los salvadoreños. $\mathrm{Ni}$ es así, ni puede serlo.

Si recordamos que Rousseau define que en el contrato social el compromiso de los individuos, como partes contratantes, es con toda la humani$\mathrm{dad}$, veremos que hay en este punto una coincidencia importante: también en el caso salvadoreño hay un compromiso de las partes con toda la comunidad internacional.

De ahí se desprende un derecho de intervención internacional, que es positivo desde el punto de vista de la paz, y, por tanto, de los intereses nacionales. También se deduce una obligación inversa: la comunidad internacional no puede desentenderse de la marcha del proceso pacificador salvadoreño, vinculada como está, moral y políticamente, por el tipo especial de contrato que supone el Acuerdo de Chapultepec. Esto vale para el período pre-electoral, como para el período postelectoral, tanto para los compromisos de tipo financiero, como para los de índole política, social, de asistencia técnica, etc.

El compromiso de Chapultepec, este tipo de contrato que por su propia naturaleza atane a toda la humanidad, tiene una faceta particularmente sensible: el informe de la Comisión de la verdad. De todos los compromisos alcanzados, éste es, sin lugar a dudas, el que con mayor razón posee una dimensión que excede el ámbito de lo salvadoreก๊o.

Lo que está en juego son principios y valores comunes a toda la humanidad -principios defendidos desde hace casi dos siglos y medio por Rousseau-, como el del imperio de la ley, el que ésta sea universal, que no admita privilegios (impunidad), etc.; lo que está en juego es si crímenes horrendos cometidos sistemáticamente a lo largo de más de una década, contra parte de la población salvadoreña, serán efectivamente tratados por la comunidad internacional como "crímenes de lesa humanidad", si serán reconocidos por la humanidad como crímenes en su contra.

Algunos que se oponen al informe han señalado que la Comisión de la verdad no puede preten- 


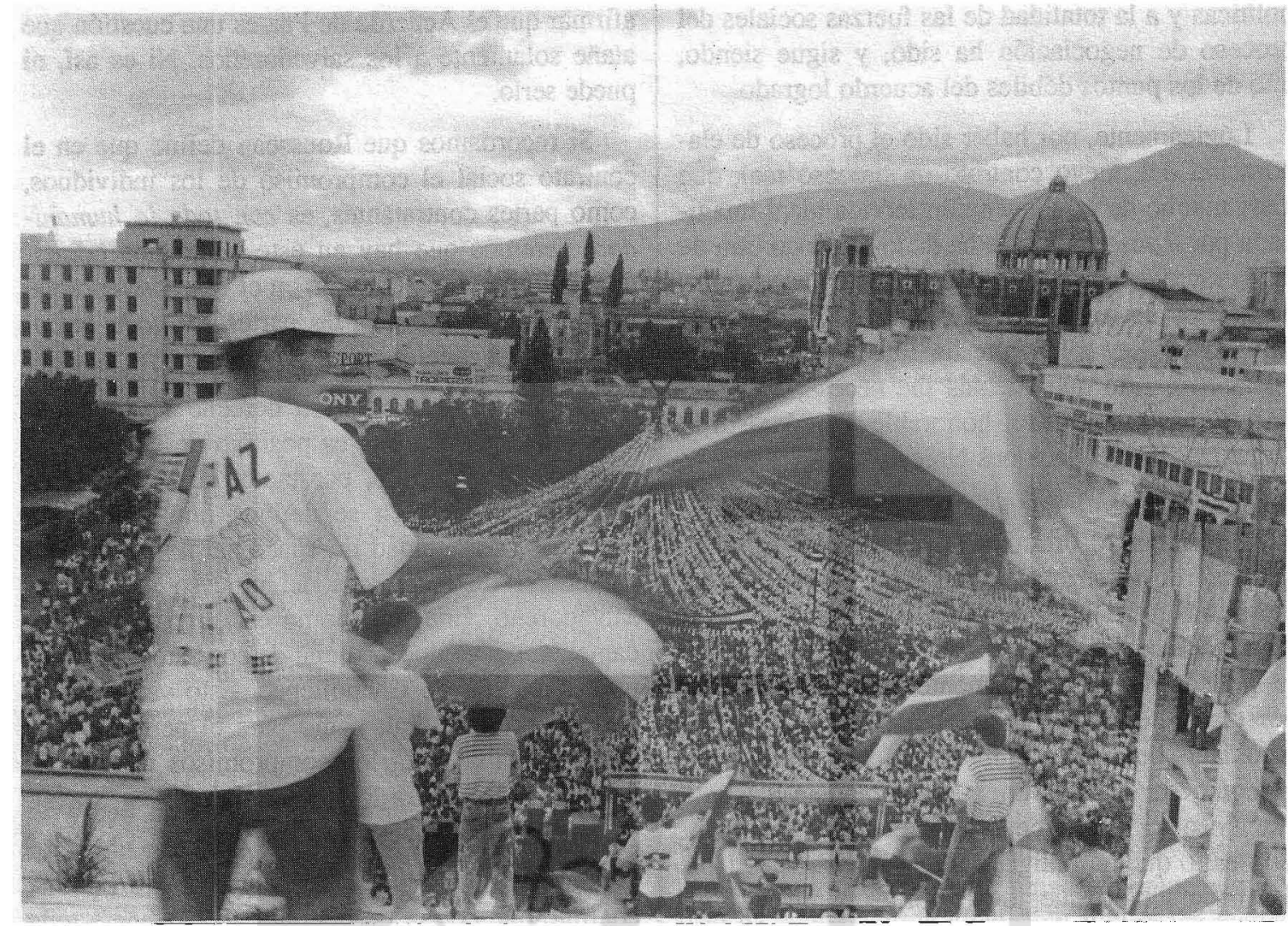

der realizar en El Salvador una especie de segundo tribunal de Nurenberg. En Nurenberg, al término de la segunda guerra mundial, como es sabido, fueron enjuiciados y condenados los criminales de guerra nazis, es decir, los vencidos. Ahí no se habló de "los excesos" cometidos por los combatientes de los países aliados, ni de los mortíferos bombardeos contra objetivos civiles en las ciudades alemanas, ni de la criminal decisión de lanzar la bomba atómica sobre dos ciudades japonesas...

Pues bien, podriamos contestar, imitando el método polemizador de Rousseau, que, efectivamente, es así; pero por razones y con consecuencias contrarias a las que con tal aseveración se pretenden. Dado que en El Salvador la solución negociada evitó que hubiera vencedores y vencidos, estamos, en realidad, en mejor situación para una aplicación más equitativa y sin distingos de los principios universales de la justicia. A diferencia del uribunal de Nurenberg, la Comisión de la verdad no sienta en el banquillo de los acusados a los vencidos; tampoco podria hacerlo puesto que no hay tales. Por tanto, la imparcialidad y universalidad de su informe es tanto mayor. La exigencia de cumplimiento efectivo de todas las recomendaciones debe entonces, asimismo, no hacer distingos sobre a cuál bando pertenece o ha pertenecido cada implicado.

Jon Sobrino ha indicado que el informe de là verdad deberia ser considerado como un documento fundante de la nueva sociedad salvadorenta. Desde un punto de vista rusoniano, la idea es totalmente correcta: el contrato social, la Constitución misma, deben estar asentados sobre un fundamento, el cual debe ser firme, sólido, imperecedero. Esta base no puede estar constituida sino por principios universales, derivados de la propia naturaleza humana, ésa que fundamenta el sentido de la dignidad del hombre. Ahora bien, ¿dónde, sino en los criterios y recomendaciones del informe de la Comisión de la verdad, están mejor concretados tales principios de justicia, principios de validez 
universal? Ni la misma Constitución debería, por tanto, poder prevalecer sobre él, dado que expresa y contiene principios que son anteriores y de naturaleza superior a cualquier derecho positivo, incluido el derecho constitucional.

De ahr que las recomendaciones de la Comisión de la verdad relativas a que se efectúen otras reformas adicionales al texto constitucional, deben ser atendidas. Asimismo, las que se refieren a la depuración profunda de la Fuerza Armada, a la investigación a fondo de las redes de escuadrones de la muerte, y a la remoción y reestructuración completa del poder judicial salvadoreño, comenzando por la Corte Suprema de Justicia. Esta, debe recordarse, es duramente señalada en el informe por su inacción, rayana en la complicidad, y por su actitud reiteradamente obstructora de las investigaciones sobre violaciones graves de los derechos humanos. La obstinación de quienes encabezan este órgano del Estado, que pretenden aferrarse a sus posiciones de poder y a la tradición de arbitrariedad que ha reinado en el sistema de justicia salvadorefío, constituye uno de los mayores obstáculos para construir un verdadero Estado de derecho en El Salvador.

El Estado de derecho es condición necesaria, aunque no suficiente, para la democracia. La primacía de la ley y la independencia del poder judicial respecto a los demás poderes son esenciales. Rousseau advertía que no debe confundirse la ley con la aplicación de la ley; Jebemos retomar esa idea, aunque en un sentido inverso a la intención rusoniana. El problema secular de El Salvador no ha sido la carencia de leyes, que las hemos tenido y algunas muy buenas, sino que ha estado centrado en su aplicación. La reflexión de Marx - a la que apunta asimismo Rousseau - de que en una sociedad desigual es injusto el derecho igual, pues en tal situación el derecho debería ser asimismo desigual, a fin de favorecer al más desprotegido, se vuelve casi irrelevante en el caso salvadoreño: aquí no sólo hemos tenido derecho igual, sino además aplicado desigualmente, ipero en contra de los más débiles!

De la lista de causas del cruento conflicto que ha devastado nuestro país, mucho se tiende a exagerar el papel de los factores económicos, olvidan- do que lo que en primera instancia mueve a los pueblos son las injusticias. No es tanto la pobreza de las mayorías - la que podría ser razonablemente aceptada en un país que es efectivamente pobre--, sino el escándalo de la ostentosa opulencia de las minorías, la que ha generado violencia. No es tanto la situación subordinada de las clases trabajadoras, sino la forma brutal con la que la clase dirigente ha ejercido su dominación, con represión cruel y con frecuencia indiscriminada, la que ha generado violencia. La injusticia generalizada, institucionalizada, sistematizada, ha sido - estamos convencidos - el primer y principal factor desencadenante de la guerra civil.

"Mientras un pueblo se ve obligado a obedecer $y$ obedece, hace bien; mas si en el momento en que puede sacudir su yugo, lo sacude, hace todavia mejor", afirmaba, radical, Rousseau. Con todo, la grandeza del pueblo salvadoreño ha consistido, no únicamente en su decisión de rebelarse ante este estado de cosas y en su capacidad de resistencia frente a la opresión, sino principalmente en su fuerza moral para dar la lucha desde ideales social y éticamente válidos. Fácilmente hubiera podido deslizarse hacia posturas cínicas, egoistas, faltas de valores morales. Existe también una forma amoral de rebelarse, ésa que reflejaba el inolvidable Maquiavelo, quien mucho antes que Rousseau fue también a su modo un inconformista con el orden existente: "le dan el falso nombre de ganancias a lo que han robado con engaños y violencia", "los siervos fieles son siempre siervos, y los hombres buenos son siempre pobres". Rousseau sentía una rara admiración por este autor florentino del siglo XVI, de quien hacía una poco usual interpretación: "Maquiavelo, fingiendo dar lecciones a los reyes, se las ha dado muy grandes a los pueblos". Sin embargo, la obra rusoniana se encaminó, contrariamente a Maquiavelo, a la conceptualización de firmes ideales éticos del hombre y de la sociedad, sobre los que sentar las bases para una sociedad alternativa.

Justamente el tipo de lazo indisoluble entre la ética y la política que está a la base de la propuesta rusoniana, debe ser retomado en El Salvador, donde la postguerra está mostrando vacilaciones y retrocesos en este campo. Han empezado a apare- 
cer claras muestras de predominio de "intereses particulares" de parte de dirigentes y partidos políticos, incluidas las fuerzas opositoras. El llamado rusoniano contra la presencia de "asociaciones particulares" en el seno del Estado, sigue siendo actual, así como su advertencia contra la defensa a ultranza de intereses particulares - bien sean éstos privados, bien sean éstos de tipo gremial o partidarista - como destructores de la "voluntad general", como manipuladores de la soberanía popular, propensos a prometer lo que después no pueden 0 no quieren cumplir. "Nunca se corrompe al pueblo -reflexionaba amargamente Rousseau- pero frecuentemente se le engaña".

Si la tendencia a despreciar las consideraciones éticas en la práctica política llegase a consolidarse, el futuro democrático en El Salvador va a sufrir una grave depreciación. Si la democracia no am- plía horizontes, si se conforma con un paisaje que se observa hoy por hoy estrictamente limitado, si no trasciende lo que es por el momento aparentemente posible, la caída en un pragmatismo y conformismo generalizado será la muerte del potencial de vitalidad democrática. En este caso, el espíritu rusoniano será más actual y necesario que nunca, su intuitiva rebeldia, que lo llevó -como afirma Agnes Heller, la erudita analista húngara"a rechazar el presente y la simiente del futuro", a rechazar un presente feudal y un futuro burgués. Parecidamente, no era tolerable en El Salvador una siluación de dictadura oligárquica y tampoco será aceptable un futuro que se limitase a las fórmulas convencionales de la democracia burguesa.

R. R.

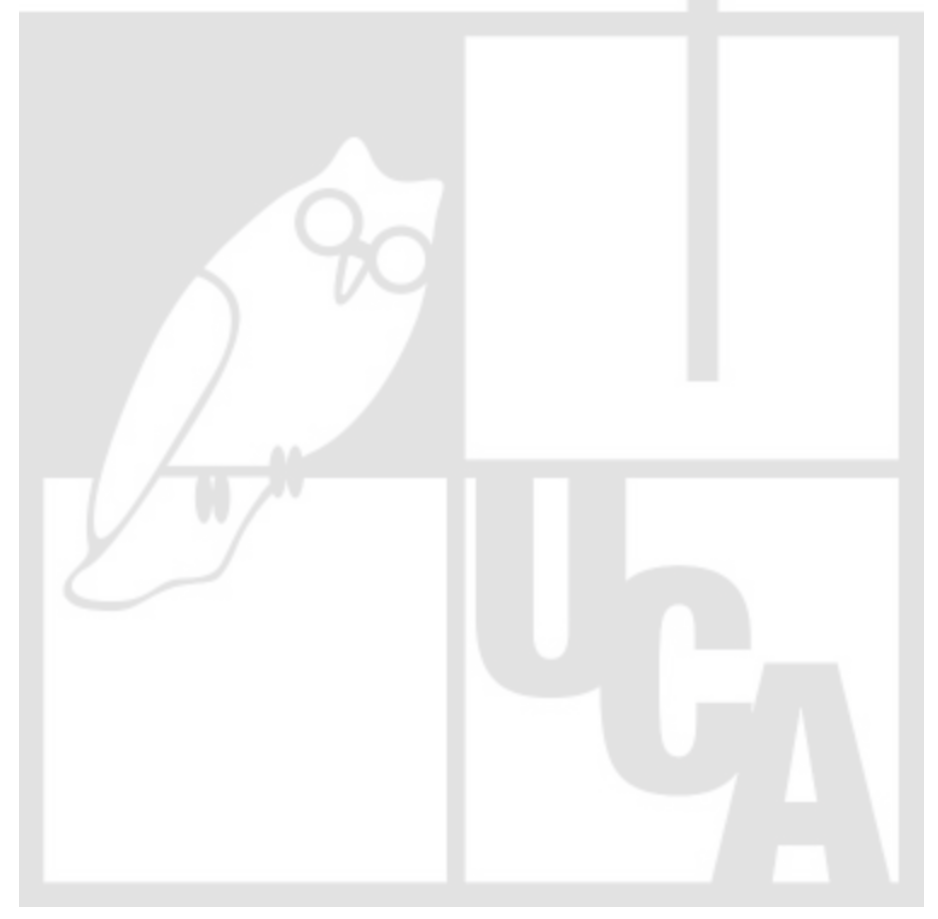

